\title{
¿Es mandatoria la tamización de disfunción tiroidea en toda mujer en la consulta preconcepcional?: Aportes de un estudio local
}

\author{
Luis Felipe Fierro Maya ${ }^{1}$, Angélica María González Clavijo², María Fernanda Garcés ${ }^{3}$, María \\ Teresa Pérez $z^{4}$ Ariel Iván Ruiz ${ }^{5}$, José Alexander Carreño ${ }^{6}$, Jorge Caminos ${ }^{7}$
}

${ }^{1}$ Instituto Nacional de Cancerología, Departamento de Endocrinología, calle $1 N^{\circ}$ 9-85 - Bogotá D.C, celular: 3006185631 , correo:angelik_md@yahoo.com

${ }^{2}$ Fellow Endocrinología, Universidad Nacional de Colombia.

${ }^{3}$ Bacterióloga, Unidad de Bioquímica, Universidad Nacional de Colombia.

${ }^{4}$ Profesora Asociada Departamento de Medicina Interna, Universidad Nacional de Colombia.

${ }^{5}$ Profesor Titular Departamento de Obstetricia y Ginecología, Universidad Nacional de Colombia.

${ }^{6}$ Epidemiólogo, Instituto Nacional de Cancerología

${ }^{7}$ Profesor Unidad de Bioquímica, Universidad Nacional de

Colombia.

Institución donde se realizó el trabajo: Universidad Nacional de

Colombia - Sede Bogotá

Autor para correspondencia: Luis Felipe Fierro Maya, correo: angelik_md@yahoo.com

\section{Resumen}

E hipotiroidismo se ha relacionado con dislipidemia, aterosclerosis, infertilidad, oligo-amenorrea y posibles efectos durante la gestación; sin embargo, hoy no hay consenso acerca de los criterios de tamización de disfunción tiroidea en la población.

Objetivo: Determinar en una muestra de mujeres de Bogotá la frecuencia de disfunción tiroidea, mediante la medición de niveles séricos de TSH y T4, para correlacionarlos con la presencia de factores de riesgo y síntomas.

Métodos: Estudio de corte transversal que incluyó una muestra de mujeres sanas entre los 16 y los 35 años de edad, a quienes se les midieron los niveles plasmáticos de TSH y T4 por electroquimioluminiscencia y se indagó sobre la presencia de síntomas y de factores de riesgo de disfunción tiroidea.

Resultados: De 186 participantes, 34 (18,3\%) presentaron disfunción tiroidea (29 tuvieron hipotiroidismo subclínico (15,60\%, IC 95\%: 10,7-21,6\%), una presentó hipotiroidismo franco $(0,53 \%$; IC 95\%: 0,0-3,0\%) y 4, hipertiroidismo subclínico $(2,15 \%$, IC $95 \%$ : 0,6-5,4\%)). No se encontraron diferencias estadísticamente significativas entre las participantes con hipotiroidismo (franco y subclínico) y aquellas con función tiroidea normal y la presencia de síntomas clínicos. La exposición a uno o más factores de riesgo no aumentó la probabilidad de presentar disfunción tiroidea (OR 1,03; IC: 0,465-2,297; p = 0,9).

Conclusión: En esta muestra transversal de mujeres se encontró una frecuencia de hipotiroidismo del 15\% en ausencia de factores de riesgo, lo que sugiere la necesidad de realizar un estudio poblacional para estimar la prevalencia de disfunción tiroidea que permita tomar una decisión de salud pública frente a la tamización universal en mujeres en edad fértil.

Palabras clave: Enfermedades de la glándula tiroides, Pruebas de función de la tiroides, Factores de riesgo en mujeres en periodo fértil.

\section{Introducción}

El hipotiroidismo, aún en rango subclínico, se encuentra asociado con diversas alteraciones en el sistema cardiovascular tales como dislipidemia( ${ }^{(1)}$, $\operatorname{arterioesclerosis}^{(2)}, \mathrm{y}$ falla cardiaca $^{(3)}$. También se ha relacionado con alteraciones en el ciclo menstrual $^{(4)}$, e infertilidad femenina ${ }^{(5)}$, así como con una mayor morbilidad perinatal, hipertensión gestacional y desprendimiento prematuro de placenta ${ }^{(6,7)}$. Por otro lado, el hipertiroidismo (franco y subclínico) se asocia con una mayor prevalencia de arritmias cardiacas $^{(8,9)}$, y una menor densidad mineral ósea ${ }^{(10,11)}$.

La prevalencia de hipotiroidismo en el estudio NANHES III $^{(12)}$ en la población méxico-americana residente en los Estados Unidos fue del 4,1\% (0,2\% para hipotiroidismo franco y 3,9\% para hipotiroidismo subclínico) y la del hipertiroidismo del $0,7 \%$ (0,3\% para el hipertiroidismo franco y 0,4\% para el hipertiroidismo subclínico). En otro estudio más reciente también realizado con población mexicana se encontró una prevalencia de 7,48\% para hipotiroidismo y de 1,7\% para hipertiroidismo subclínico y franco ${ }^{(13)}$.

Las publicaciones sobre prevalencia de disfunción tiroidea en Colombia son escasas, pero sugieren una mayor frecuencia 
de hipotiroidismo que en otras poblaciones. Por ejemplo, en 1994 en la ciudad de Cúcuta (Norte de Santander) ${ }^{(14)}$ se encontró una frecuencia de hipotiroidismo del 7,7 \% (IC 95\%: 5,7$14,4 \%$ ) sin que se discriminara entre hipotiroidismo franco y subclínico. En otro estudio, realizado en el Hospital Militar Central de Bogotá(15), que incluyó pacientes de ambos sexos sin diagnóstico previo de disfunción tiroidea, se encontraron niveles de TSH entre 4 y $10 \mu \mathrm{UI} / \mathrm{mL}$ en el $24,41 \%$ de los pacientes y se halló TSH mayor a $10 \mu \mathrm{UI} / \mathrm{mL}$ en el 7,4\%; el 4,95\% tuvo una TSH suprimida menor de $0,4 \mu \mathrm{UI} / \mathrm{mL}$ y el resto de los pacientes tuvo una TSH normal.

Con respecto a los criterios de tamización de disfunción tiroidea en la población general y mujeres embarazadas aún no existe consenso. La guía del Grupo de Trabajo de Servicios Preventivos de los Estados Unidos (US Preventive Services Task Force, USPSTF) para la tamización de disfunción tiroidea subclínica en adultas no embarazadas ${ }^{(16)}$ concluye que es incierto el beneficio de la tamización universal, en términos de costo-efectividad. Por otro lado, la guía de práctica clínica de la Sociedad Americana de Endocrinología (American Endocrine Society), sobre el tratamiento de la disfunción tiroidea durante el embarazo y el posparto ${ }^{(7)}$ recomienda que el hipotiroidismo clínico y subclínico deben ser reconocidos y corregidos antes del inicio del embarazo, pero no se recomienda la tamización universal, sino limitada a las embarazadas que tengan uno o más factores de riesgo, tales como: enfermedad tiroidea previa, bocio, uso de medicamentos de acción tiroidea, antecedente de radiación cervical, antecedente de tratamientos con yodo radioactivo, cirugía tiroidea previa o presencia de anticuerpos antitiroideos. Sin embargo, el valor predictivo de tales factores no ha sido estudiado en ensayos prospectivos a largo plazo y, además, la prevalencia de disfunción tiroidea parece seguir siendo importante aun cuando se excluyen los pacientes con algunos de los factores de riesgo mencionados ${ }^{(12)}$.

El objetivo de esta investigación fue determinar el diagnóstico de disfunción tiroidea mediante la medición de niveles séricos de TSH y T4 en una muestra de mujeres jóvenes, aparentemente sanas, en diferentes instituciones de la ciudad de Bogotá, y correlacionar los resultados con la presencia de síntomas y factores de riesgo que tradicionalmente han sido atribuidos a las enfermedades de la tiroides.

\section{Materiales y métodos}

El diseño del estudio fue de corte transversal. Para la inclusión de las participantes se realizó una invitación abierta a mujeres jóvenes entre los 16 y 35 años de edad, a través del correo electrónico y anuncios en las facultades de medicina, enfermería y nutrición de la Universidad Nacional de Colombia, el colegio Distrital Miguel Antonio Caro, la Clínica Fundadores y el Hospital Universitario Clínica San Rafael de Bogotá, entre el 1 de marzo y el 1 de septiembre de 2007.
Para ser incluidas en el estudio, las mujeres deberían no estar en embarazo o lactancia y otorgar voluntariamente consentimiento informado para participar. Se excluyeron mujeres con enfermedad tiroidea activa o que estuvieran recibiendo tratamiento en los últimos seis meses con levotiroxina, metimazol, propiltiouracilo, amiodarona y carbonato de litio. No se tuvieron en cuenta los antecedentes quirúrgicos.

Se diseñó una encuesta semiestructurada para indagar en la muestra de participantes los signos y síntomas, así como los factores de riesgo para enfermedad tiroidea (Anexo 1). Posteriormente se hizo una prueba piloto con 20 participantes y se ajustaron las preguntas.

La recolección y análisis de las muestras de las participantes se realizó en el laboratorio del Departamento de Bioquímica de la Universidad Nacional de Colombia, las participantes deberían cumplir con las siguientes condiciones necesarias para la toma de la muestra: tener ayuno de 8 horas, no haber ingerido licor o haber fumado 8 horas antes y no haberse tinturado el cabello en los 8 días previos a la toma de las muestras sanguíneas. Las muestras fueron obtenidas por punción venosa de $5 \mathrm{~mL}$ de sangre, los plasmas fueron separados por centrifugación y congelados a - 20 grados centígrados de manera inmediata. Posteriormente fueron procesadas en conjunto para determinar los niveles plasmáticos de TSH y T4, mediante electroquimioluminiscencia en el analizador automático Roche Elecsys 2010 (Roche Diagnostics GmbH), del laboratorio de Unisalud (Bogotá).

Los datos obtenidos fueron almacenados en una base de datos, usando la hoja de cálculo de Microsoft ${ }^{\circledR}$ y analizados con el paquete estadístico de STATA versión 8,0.

El análisis estadístico incluyó descripción de variables mediante el cálculo de medidas de frecuencia absoluta y relativa en el caso de las variables cualitativas. Para las variables cuantitativas, se resumió la información con medidas de tendencia central (promedios o medianas) y medidas de dispersión (desviaciones estándar o rangos), acorde con la normalidad de la distribución. Se validó el supuesto de normalidad y se realizó un análisis bivariado para establecer la asociación entre los factores de riesgo y el diagnóstico de disfunción tiroidea mediante el cálculo de Odds Ratio (OR).

\section{Resultados}

Se incluyeron 186 participantes que cumplieron los criterios de inclusión. Dentro de las características de la población estudiada se encontró que el promedio de edad fue de 22,6 años $\pm 4,9$. El $81,2 \%$ había tenido relaciones sexuales y el $30,1 \%$ había tenido al menos un embarazo. Diez participantes manifestaron haber presentado al menos un aborto espontáneo $(10,2 \%)$. El $27 \%$ de las mujeres sexualmente activas (41 de 151) manifestaron que llevaban al menos un año de infertilidad. La frecuencia de uso de anticonceptivos hormonales fue 
Tabla 1. Características de las 155 mujeres en edad fértil de acuerdo con las determinaciones de TSH y T4 libre

\begin{tabular}{|c|c|c|c|c|c|}
\hline & \multicolumn{5}{|c|}{ Interpretación valores TSH y T4 } \\
\hline & Normal & $\begin{array}{c}\text { Anormal } \\
\text { hipertiroidismo } \\
\text { subclínico }\end{array}$ & $\begin{array}{l}\text { Anormal } \\
\text { hipotiroidismo } \\
\text { subclínico }\end{array}$ & $\begin{array}{c}\text { Anormal } \\
\text { hipotiroidismo }\end{array}$ & Total \\
\hline Frecuencia & $155(83,3 \%)$ & $1(0,5 \%)$ & $29(15,6 \%)$ & $1(0,5 \%)$ & 186 \\
\hline Promedio de edad & 22,4 & 26 & 23,5 & 23 & --- \\
\hline \multicolumn{6}{|l|}{ Antecedentes } \\
\hline Enfermedad tiroidea previa & $2(1,1 \%)$ & 0 & $2(1,1 \%)$ & 0 & 4 \\
\hline Enfermedad autoinmune & $3(1,6 \%)$ & 0 & 0 & 0 & 3 \\
\hline Antecedentes familiares & $13(7 \%)$ & 0 & $5(2,7 \%)$ & 0 & 18 \\
\hline Historia de infertilidad & $38(20,4 \%)$ & 0 & $3(1,6 \%)$ & 0 & 41 \\
\hline Inicio menarquia & 12,5 & 13 & 13,2 & 12 & --- \\
\hline Antecedentes de aborto & $15(8,1 \%)$ & $1(0,5 \%)$ & $3(1,6 \%)$ & 0 & 19 \\
\hline \multicolumn{6}{|l|}{ Signos y síntomas } \\
\hline Caída de cabello & $60(32,3 \%)$ & $1(0,5 \%)$ & $14(7,5 \%)$ & 0 & 75 \\
\hline Casancio inexplicado & $64(34,4 \%)$ & $1(0,5 \%)$ & $10(5,4 \%)$ & 0 & 75 \\
\hline Cambios de peso & $42(22,6 \%)$ & $1(0,5 \%)$ & $9(4,8 \%)$ & 0 & 52 \\
\hline Irregularidad menstrual & $107(57,5 \%)$ & 0 & $20(10,8 \%)$ & 0 & 127 \\
\hline Estreñimiento & $26(14 \%)$ & $1(0,5 \%)$ & $4(2,2 \%)$ & 0 & 31 \\
\hline Intolerancia a la temperatura & $55(29,6 \%)$ & $1(0,5 \%)$ & $8(4,3 \%)$ & 0 & 64 \\
\hline Palpitaciones & $35(18,8 \%)$ & $1(0,5 \%)$ & $4(2,2 \%)$ & 0 & 40 \\
\hline
\end{tabular}

del 22,5\%, pero no hubo diferencias en la frecuencia de alteraciones de los valores de TSH entre las usuarias de anticonceptivos frente a las no usuarias. Con relación a los síntomas de disfunción tiroidea, los más frecuentes fueron la caída del cabello (75 mujeres, 40,3\%) y el cansancio fácil, (75 mujeres, 40,3\%). El 31,7\% de las encuestadas manifestó tener ciclos menstruales irregulares (menores a 25 días o mayores a 35). Sólo el 30\% de las participantes reportó estar libre de los síntomas investigados (tabla 1).

Usando un punto de corte de TSH mayor de 4,2 para definir hipotiroidismo y menor de $0,2 \mathrm{uUI} / \mathrm{mL}$ para definir hipertiroidismo, el $18,28 \%$ de las mujeres estudiadas presentaron anormalidades en la función tiroidea, distribuidas de la siguiente manera: 29 tuvieron hipotiroidismo subclínico (15,60\%; IC95\%: 10,7-21,6\%), una tuvo hipotiroidismo franco (0,53\%; IC 95\%: 0,0-,0\%) y 4 mujeres hipertiroidismo subclínico $(2,15 \%$, IC 95\%: 0,6-5,4\%). Entre las mujeres con TSH en el rango normal, el percentil 50 de TSH fue de $1,86 \mu \mathrm{UI} / \mathrm{mL}$, con un promedio de $2,0 \mu \mathrm{UI} / \mathrm{mL} \pm 0,85$. El percentil 50 de TSH
Tabla 2. Resultados de TSH en las 155 mujeres en edad fértil, agrupados en rangos de supresión $(<0,2 \mu \mathrm{UI} / \mathrm{mL})$, normalidad $(0,2$ a 4,2 $\mu \mathrm{UI} / \mathrm{mL}$ ) e hipotiroidismo (mayor a $4,2 \mu \mathrm{UI} / \mathrm{mL}$ y mayor o igual a $10 \mu \mathrm{UI} / \mathrm{mL}$ )

\begin{tabular}{l|c|c}
\multicolumn{1}{c|}{ TSH } & Frecuencia & Porcentaje \\
\hline$<0,2 \mu \mathrm{UI} / \mathrm{mL}$ & 4 & $2,20 \%$ \\
\hline 0,2 a $4,2 \mu \mathrm{UI} / \mathrm{mL}$ & 152 & $81,70 \%$ \\
\hline 4,21 a $9,99 \mu \mathrm{UI} / \mathrm{mL}$ & 27 & $14,50 \%$ \\
\hline$\geq 10,00 \mu \mathrm{UI} / \mathrm{mL}$ & 3 & $1,61 \%$ \\
\hline Total & $\mathbf{1 8 6}$ & \\
\hline
\end{tabular}

en los casos de hipotiroidismo subclínico fue de 5,26 $\mu \mathrm{UI} / \mathrm{mL}$, con un rango entre 4,26 y 17,94 $\mu \mathrm{UI} / \mathrm{mL}$ (tabla 2).

La distribución de los valores de tiroxina sérica (T4) fue: el percentil 50 fue de 7,65 $\mu \mathrm{g} / \mathrm{dL}$, con un valor promedio de 7,89 $\mu \mathrm{g} / \mathrm{dL} \pm 1,4 \mathrm{DE}$. Sólo una paciente tuvo un valor por fuera del rango normal (tabla 3 ). 
Tabla 3. Resultados de T4 total en las 155 mujeres en edad fértil, agrupados según los rangos de referencia del laboratorio: baja $(<5,13 \mu \mathrm{g} / \mathrm{dL})$, normal $(5,13 \mathrm{a} 14 \mu \mathrm{g} /$ dL) y alta (mayor a 14,06 $\mu \mathrm{g} / \mathrm{dL}$ )

\begin{tabular}{l|c|c}
\multicolumn{1}{c|}{ T4 $\mu \mathbf{g} / \mathbf{d L}$} & Frecuencia & Porcentaje \\
\hline$<5,13$ & 1 & $0,54 \%$ \\
\hline 5,13 a 14,6 & 185 & $99,46 \%$ \\
\hline$>14,06$ & 0 & $0 \%$ \\
\hline
\end{tabular}

Tabla 4. Ausencia de asociación entre los factores de riesgo y la presencia de disfunción tiroidea

\begin{tabular}{l|c|c|c}
\hline \multicolumn{1}{c|}{ Antecedentes } & Frecuencia & OR (IC 95\%) & p \\
\hline Enfermedad tiroidea previa & $4(2,1 \%)$ & $5,35(0,725-39,6)$ & 0,25 \\
\hline Enfermedad autoinmune & $3(1,6 \%)$ & $0,7(0,035-13,9)$ & 0,66 \\
\hline Antecedentes familiares & $18(9,7 \%)$ & $2,13(0,7-1,46)$ & 0,3 \\
\hline Historia de infertilidad & $41(22 \%)$ & $0,33(0,09-1,16)$ & 0,11 \\
\hline Antecedentes de abortos & $19(10,2 \%)$ & $1,015(0,275-3,78)$ & 0,75 \\
\hline
\end{tabular}

Tabla 5. OR para TSH elevada o suprimida en relación con la presencia de signos y síntomas

\begin{tabular}{l|c|c|c}
\hline \multicolumn{1}{c|}{ Signos y síntomas } & Frecuencia & OR (IC 95\%) & p \\
\hline Caída de cabello & $75(40,7 \%)$ & $1,21(0,57-2,56)$ & 0,61 \\
\hline Cansancio inexplicado & $75(40,7 \%)$ & $0,77(0,35-1,67)$ & 0,5 \\
\hline Cambios de peso & $52(27,9 \%)$ & $1,09(0,48-2,47)$ & 0,83 \\
\hline Irregularidad menstrual & $126(67,7 \%)$ & $0,52 \%(0,21-1,29)$ & 0,16 \\
\hline Estreñimiento & $31(16,6 \%)$ & $0,83(0,29-2,36)$ & 0,73 \\
\hline
\end{tabular}

Respecto a los factores de riesgo reportados, un total de 90 $(45,6 \%)$ participantes reportaron tener al menos una condición considerada como factor de riesgo de disfunción tiroidea, no obstante, la exposición a uno o más factores de riesgo no aumentó la probabilidad de presentar disfunción tiroidea (OR 1,03; IC: $0,465-2,297 ; p=0,9$ ) y se encontró hipotiroidismo (franco y subclínico) en el 12,5\% (12 de 96) de las mujeres sin ningún factor de riesgo (tabla 4).

La presencia de síntomas tradicionalmente atribuidos a disfunción tiroidea y específicamente a hipotiroidismo fue frecuente entre las participantes, sobre todo la irregularidad menstrual $(67,7 \%)$, caída de cabello $(40,7 \%)$ y cansancio fácil $(40,7 \%)$ pero no se encontraron diferencias estadísticamente significativas entre las participantes con TSH elevada y aquellas con TSH en rango normal (tabla 5).

\section{Discusión}

En este trabajo se incluyeron mujeres entre los 16 y los 35 años, rango que comprende la edad promedio a la cual las mujeres colombianas tienen su primer hijo $(21,6 \text { años })^{(17)}$ confirién- dole relevancia desde los puntos de vista clínico, epidemiológico y de salud pública por cuanto los efectos del hipotiroidismo aún en el rango subclínico, pueden ser más deletéreos que en otros grupos poblacionales.

La frecuencia de hipotiroidismo subclínico en esta muestra fue mucho mayor que la reportada en el estudio NANHES III ${ }^{(12)}$ para las mujeres méxico-americanas aparentemente sanas, no embarazadas, a pesar de usar el mismo punto de corte $(>4,5$ $\mu \mathrm{UI} / \mathrm{mL})$ para TSH $(5,3 \%$ vs. $13,4 \%)$. La prevalencia de TSH $>5,0 \mu \mathrm{UI} / \mathrm{mL}$ encontrada en esta muestra también fue más alta que la reportada en el estudio de la ciudad de Cúcuta ${ }^{(14)}$ ( 7,7 vs. $10,2 \%$ ), a pesar de que el promedio de edad en esta investigación fue menor (46,3 vs. 22,6 años). Se desconoce la existencia de otros factores regionales que justifiquen esta diferencia, pues en otro estudio en la ciudad de Bogotá también se reportó una frecuencia mayor de hipotiroidismo ${ }^{(15)}$. Aunque al comparar los resultados que obtuvimos con los de este último estudio, la prevalencia de TSH entre 4,0 y $10 \mu \mathrm{UI} / \mathrm{mL}$ fue más baja (15,0\% vs. 24,9\%). Sin embargo, la diferencia podría explicarse porque nuestra población fue más joven (promedio de edad 22,6 vs. 52 años) y también porque en ese estudio el $12,52 \%$ de la población de mujeres estuvo entre 18 y 30 años.

Con respecto a la importancia de los factores de riesgo de disfunción tiroidea como criterio para seleccionar a las pacientes que deben ser tamizadas para detectar tal alteración, nuestros resultados fueron concordantes con los reportados por Vaidya y colaboradores ${ }^{(18)}$, en el sentido de que la presencia de factores de riesgo no fue estadísticamente diferente entre las participantes con hipotiroidismo (franco y subclínico) $\mathrm{y}$ aquellas con función tiroidea normal. A diferencia de dicho estudio, la frecuencia de hipotiroidismo en mujeres sin factores de riesgo encontrada por nosotros fue más baja (12,5\% vs. $30 \%)$. Esta diferencia puede ser causada porque la población incluida por Vaidya fue de mujeres embarazadas.

En contraste, nuestros hallazgos fueron bastante diferentes a los publicados en el estudio NAHNES III ${ }^{(12)}$, en el cual la prevalencia de $\mathrm{TSH}>4,5 \mathrm{uUI} / \mathrm{mL}$ en el subgrupo de mujeres méxico-Americanas no embarazas y sin factores de riesgo de disfunción tiroidea, fue de sólo el 2,2\% $\pm 0,5$.

En los resultados que obtuvimos no se encontraron diferencias estadísticas entre la presencia de síntomas en las mujeres con hipotiroidismo y aquellas con TSH normal. Esto sugiere que el tamizaje guiado por síntomas debería ser reevaluado, al menos en mujeres jóvenes, dado que tales síntomas no son específicos, son muy frecuentes en la población y no predicen disfunción tiroidea.

Dado que las diferentes guías sobre control prenatal ${ }^{(7,19)}$ mencionan la importancia de reconocer y corregir el hipotiroidismo clínico y subclínico antes del inicio del embarazo, debido a sus posibles efectos sobre el desarrollo neuropsicológico y supervivencia del feto, y su asociación con toxemia e hipertensión, nuestros resultados apuntan hacia la promoción de 
la tamización universal de mujeres en edad fértil que tengan planes de embarazarse en un futuro inmediato, en contra de la recomendación actual de tamización limitada a las mujeres con factores de riesgo. Para esto se requieren estudios controlados y con poblacionales más grandes que permitan análisis ampliados que conlleven a la toma de decisiones al respecto.

Finalmente, reconocemos las limitaciones de esta investigación, como el hecho de tratarse de una medición puntual que, teniendo en cuenta la variabilidad en las mediciones de TSH y T4 total, podrían alterar la prevalencia de hipotiroidismo franco y subclínico. Tampoco tuvimos en cuenta la determinación de los títulos de anticuerpos antitiroideos que contribuirían a estratificar mejor a las mujeres en riesgo de disfunción tiroidea durante el embarazo. Recomendamos que estos resultados sean interpretados en el contexto local o regional y no pueden ser extrapolados a otros grupos poblacionales.

\section{Conclusiones}

En esta muestra transversal de mujeres se encontró una prevalencia de disfunción tiroidea del 18,28\%, siendo el hipotiroidismo subclínico la alteración más frecuente $(15,6 \%)$.
Esta frecuencia es tres veces mayor a la prevalencia encontrada en mujeres méxico-americanas no embarazadas del estudio NANHES III. No encontramos una asociación entre la presencia de factores de riesgo y los resultados de las pruebas de función tiroidea y tampoco hubo asociación con la presencia de los síntomas indagados, por lo cual consideramos que nuestros resultados apuntan hacia la tamización universal de las mujeres en edad fértil con planes de embarazo como parte de la evaluación previa al embarazo, dadas las implicaciones del hipotiroidismo en la gestación; no obstante reconocemos que es necesario realizar un estudio poblacional a mayor escala que permita tomar una decisión de salud pública frente a este tema.

\section{Declaración de interés}

Los autores declaran que no existen conflictos de interés que pudieran ser percibidos como perjudiciales para la imparcialidad del artículo.

\section{Financiación}

No se recibió ninguna fuente externa de financiación diferente a los recursos propios aportados por los investigadores.

\section{Anexo 1}

\section{Encuesta de tamizaje de disfunción tiroidea}

La información aquí solicitada es requerida como parte del trabajo de investigación de la Universidad Nacional de Colombia ${ }^{\S}$, cuyo objetivo general es determinar la frecuencia de alteraciones de la función de la glándula tiroides en mujeres jóvenes, no embarazadas.

Por favor, conteste las siguientes preguntas de acuerdo con su situación personal. Se garantiza total reserva de la información aquí registrada.

\section{Datos de identificación:}

Fecha: Edad:__ Identificación:

\section{Antecedentes personales:}

¿Sufre o ha sufrido alguna de las siguientes enfermedades? (Escriba Sí o No en el espacio en blanco)

- Hipotiroidismo _ ¿Está en tratamiento actualmente o en los últimos 6 meses?

- Hipertiroidismo _ ¿Está en tratamiento actualmente o en los últimos 6 meses?

- Tiroiditis (inflamación de la glándula tiroides)

- Bocio (tiroides aumentada de tamaño)

- Diabetes mellitus tipo 1 (requiere insulina)

- Diabetes insípida (déficit de hormona antidiurética)

- Vitiligo

- Miastenia Gravis

- Lupus eritematoso sistémico, artritis reumatoide u otras enfermedades del colágeno

- Enfermedad celíaca o esprue (diarrea crónica - malabsorción intestinal)

- Hepatitis autoinmune (no causada por los virus de la hepatitis)

- Insuficiencia adrenal o suprarrenal (requiere tomar corticoesteroides)

- Hipoparatiroidismo (alteración en el calcio sanguíneo, por falta de la hormona PTH o paratohormona) _

- Anemia crónica (con excepción de anemia por sangrado o déficit de hierro)

- Colesterol total mayor de $200 \mathrm{mg} / \mathrm{dl}$ o recibe tratamiento para disminuirlo

$\bar{\S}$ Responsable: Angélica González C, Médico. U. Nacional (Cel: 30021174 63), Dr. Felipe Fierro M, Médico U. Nacional (Cel: 300 618 5631 ) 


\section{Antecedentes ginecológicos:}

- Edad de primera menstruación: __ años

- ¿Ha tenido relaciones sexuales? Sí _ No_. Si la respuesta es No, pase a la pregunta 8 de éste punto

- ¿Ha estado en embarazo alguna vez? Sí__ No__. Si la respuesta es no, pase a la pregunta 7 de éste punto

- ¿Cuántas veces ha estado en embarazo? 1_2 _ más de 2

- ¿Ha tenido abortos espontáneos (pérdidas de la gestación antes de la semana 20 de gestación)? Sí__ No _

- ¿Ha tenido pérdidas de embarazos después de la semana 20 de gestación? Sí _ No_

- ¿Ha tenido relaciones sexuales sin métodos anticonceptivos (preservativo, píldoras, inyectables, dispositivos, implantes) durante más de un año sin quedar en embarazo? Sí__ No _

- ¿En el último año sus periodos menstruales han sido regulares (el intervalo entre 2 menstruaciones va entre 25 y 35 días): Sí_ No

- ¿Está recibiendo anticonceptivos orales o inyectables actualmente (últimos 3 meses)? Sí__ No__

\section{Antecedentes quirúrgicos:}

- ¿Le han realizado alguna cirugía en la tiroides? Sí _ _ No _ ¿Cuál cirugía?

- ¿Le han realizado cirugía de la glándula hipófisis (cerebro)? Sí _ No _ ¿Cual cirugía?

- ¿Le han realizado radioterapia en cuello, parte superior del tórax o la cabeza? Sí__ No _

- ¿Ha recibido tratamiento con yodo radioactivo? Sí_ No _

- ¿Fuma habitualmente? Sí _ No _

5. ¿Presenta alguno de los siguientes síntomas, que haya comenzado y estén presentes EN LOS ÚLTIMOS 6 MESES?

- Caída abundante del cabello (no explicada por otras enfermedades o tratamientos). Sí__ No_

- Intolerancia o sensación de frío o calor no proporcional a la temperatura del ambiente. Sí__ No_

- Cansancio crónico no explicado por alteraciones en el sueño, exceso de actividad física o estrés. Sí__ No__

- Estreñimiento (menos de 3 deposiciones a la semana) de inicio reciente. Sí_ No_

- Ganancia o pérdida de peso no explicada por dieta o ejercicio. Sí__ No__

- Palpitaciones (sentir los latidos cardiacos más fuertes o rápidos de lo usual). Sí__ No__

\section{Antecedentes familiares:}

- ¿Algún familiar en primer grado (papá, mamá o hermanos) sufre o ha sufrido de enfermedades de la tiroides? Sí _ No Cuál enfermedad:

\section{Referencias}

1. O’Brien T, Dinneen SF, O'Brien PC, Palumbo PJ. Hyperlipidemia in patients with primary and secondary hypothyroidism. Mayo Clin Proc. 1993;68:860-6.

2. Hak a. E, Pols H a. P, Visser TJ, Drexhage $H$ a., Hofman A, Witteman JCM. Subclinical Hypothyroidism Is an Independent Risk Factor for Atherosclerosis and Myocardial Infarction in Elderly Women: The Rotterdam Study. Ann Intern Med. 2000;132(4):270.

3. Biondi B, Fazio S, Palmieri EA, Carella C, Panza N, Cittadini A, et al. Left Ventricular Diastolic Dysfunction in Patients with Subclinical Hypothyroidism. 1999;84(6):2064-7.

4. Krassas GE, Pontikides N, Kaltsas T, Papadopoulou P, Paunkovic J, Paunkovic $\mathrm{N}$, et al. Disturbances of menstruation in hypothyroidism. Clin Endocrinol (Oxf). 1999;50:655-9.

5. Abalovich M1, Mitelberg L, Allami C, Gutierrez S, Alcaraz G, Otero P LO. Subclinical hypothyroidism and thyroid autoimmunity in women with infertility. Gynecol Endocrinol. 2007;23(5):279-83.

6. Neto LV, De Almeida CA, Da Costa SM, Vaisman M. Prospective evaluation of pregnant women with hypothyroidism: Implications for treatment. Gynecol Endocrinol. Informa Clin Med; 2007;23(3):138-41.

7. De Groot L, Abalovich M, Alexander EK, Amino N, Barbour L, Cobin RH, et al. Management of Thyroid Dysfunction during Pregnancy and Postpartum: An Endocrine Society Clinical Practice Guideline. J Clin Endocrinol Metab. The Endocrine Society; 2012;97(8):2543-65.

8. Sawin CT, Geller A, Wolf PA, Belanger AJ, Baker E, Bacharach P, et al. Low Serum Thyrotropin Concentrations as a Risk Factor for Atrial Fibrillation in Older Persons. N Engl J Med. Massachusetts Medical Society; 1994;331(19):1249-52.

9. Auer J, Scheibner P, Mische T, Langsteger W, Eber O, Eber B. Subclinical hyperthyroidism as a risk factor for atrial fibrillation. Am Heart J. Elsevier: 2014;142(5):838-42.

10. Kumeda Y1, Inaba M, Tahara H, Kurioka Y, Ishikawa T, Morii H NY. Persistent increase in bone turnover in Graves' patients with subclinical hyperthyroidism. J Clin Endocrinol Metab. 2000;85(11):4157-61.
11. Lastra G, Caicedo Henry. Efectos de la hormona tiroidea en dosis fisiológicas sobre la masa ósea en pacientes con hipotiroidismo subclínico. Acta Med Colomb 2000;25: 188-194.

12. Hollowell JG, Staehling NW, Flanders WD, Hannon WH, Gunter EW, Spencer $\mathrm{C}$ a, et al. Serum TSH, T(4), and thyroid antibodies in the United States population (1988 to 1994): National Health and Nutrition Examination Survey (NHANES III). J Clin Endocrinol Metab. 2002;87(2):489-99.

13. Robles-Osorio ML1, Zacarías-Rangel V, García-Solís P, Hernández-Montiel HL, Solís JC SE. Prevalence of thyroid function test abnormalities and antithyroid antibodies in an open population in Central México. Rev Invest Clin. 2014;66(2):113-20.

14. García H, Duque A SM. Frecuencia de diabetes, hipotiroidismo, obesidad e hipertensión arterial en población abierta de la ciudad de Cúcuta. Rev Clon, Univ Pamplona. 2003;74-8.

15. Builes CA, Rosero O, García J. Evaluación de disfunción tiroidea según TSH en una población de Bogotá . TSH levels in the evaluation of thryroid disfunction in a population group in Bogota. Acta Medica Colomb. 2006;31(2):66-70.

16. Helfand M. Screening for Subclinical Thyroid Dysfunction in Nonpregnant Adults: A Summary of the Evidence for the U.S. Preventive Services Task Force. Ann Intern Med. 2004;140(2):128-41.

17. Fecundidad de las mujeres [Internet]. Encuesta Nacional de Demografia y Salud - ENDS. 2010 [cited 2013 Sep 10]. p. 1 - 2. Available from: http:// www.profamilia.org.co/encuestas/Profamilia/Profamilia/documentos/ boletines/prensa/Fecundidad_de_las_Mujeres.pdf

18. Vaidya B, Anthony S, Bilous M, Shields B, Drury J, Hutchison S, et al. Detection of Thyroid Dysfunction in Early Pregnancy: Universal Screening or Targeted High-Risk Case Finding? J Clin Endocrinol Metab. The Endocrine Society; 2007;92(1):203-7.

19. Cancino E, León H, Otálora R, Pérez González E, Sarmiento R, Yates A. Guía de control prenatal y factores de riesgo. Secretaría Distrital de Salud de Bogotá, D. C. Asociación Bogotana de Obstetricia y Ginecología (Asbog). Tomada de internet, consula realizada el 1 de septiembre de 2014: http:// es.scribd.com/doc/56188953/Guia-1-Control-Prenatal-y-Factores-deRiesgo\#scribd 